\title{
GENERATION AND CONTROL OF HIGH PRECISION BEAMS AT LEPTON ACCELERATORS
}

\author{
Yu-Chiu Chao, TJNAF, Newport News 23606, U.S.A.*
}

\section{Abstract}

Parity violation experiments require precision manipulation of helicity-correlated beam coordinates on target at the $\mathrm{nm} / \mathrm{nrad}$-level. Achieving this unprecedented level of control requires a detailed understanding of the particle optics and careful tuning of the beam transport to keep anomalies from compromising the design adiabatic damping. Such efforts are often hindered by machine configuration and instrumentation limitations at the low energy end. A technique has been developed at CEBAF including high precision measurements, Mathematicabased analysis for obtaining corrective solutions, and control hardware/software developments for realizing such level of control at energies up to $5 \mathrm{GeV}$.

\section{OVERVIEW}

Recent progress at Jefferson Lab in achieving beam quality specs satisfying stringent demands set by parity violation experiments at the CEBAF accelerator is discussed in this report. While successful delivery of said beam quality specs comes as a result of efforts on multiple fronts, this report will focus on the aspect of optical transport corrections realizing adiabatic damping, which is dictated by simple dynamics, but severely obscured by errors in beam transport.

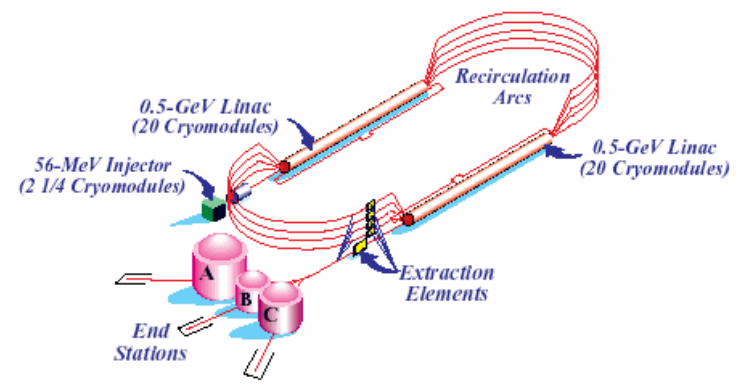

Figure 1: Schematic of CEBAF.

The CEBAF accelerator, as shown schematically in Figure 1, is a recirculating linac, delivering $\mathrm{CW}$ electron beam up to $6 \mathrm{GeV}$, to 3 fixed target experiments. It is devoted to the study of nucleon structure and quark-gluon dynamics. Electron beam, after generation and initial formation in the Injector, is transported through a $6 \mathrm{~km}$ racetrack, up to 5 times through each of the two accelerating linacs. It undergoes a complicated set of longitudinal and transverse gymnastics before arriving at the experimental targets.

\section{PARITY EXPERIMENTS AT CEBAF}

A very important and demanding class of experiments

* Notice: Authored by The Southeastern Universities Research Association, Inc. under U.S. DOE Contract No. DE-AC05-84ER40150. The U.S. Government retains a non-exclusive, paidup, irrevocable, world-wide license to publish or reproduce this manuscript for U.S. Government purposes. conducted at CEBAF is the parity violation (PV) experiment, or parity experiment, devoted to the study of fine asymmetry in elastic electron-nucleon scattering by electromagnetic and weak interactions. The electron beam is polarized in either positive or negative handedness (helicity state), and the asymmetry, $\boldsymbol{A}_{P V}$, in scattering cross sections, $\sigma_{L, R}$, between left handed \& right handed helicity states is measured:

$$
A_{P V}=\frac{\sigma_{R}-\sigma_{L}}{\sigma_{R}+\sigma_{L}} \approx \frac{Q^{2}}{M_{z}^{2}} \approx 10^{-6}-10^{-4} \text { or } 1-100 \mathrm{ppm}
$$

where $Q^{2}$ is the 4-momentum transfer and $M_{Z}$ the mass of the Z-boson. In the above we noted the typical magnitude of such asymmetry to be in the range of 1 to 100 part-per-million (ppm).

To confidently constrain the physical implications with this asymmetry, the statistical and systematic errors must be controlled to a few percent of the asymmetry itself, or to sub-100 part-per-billion (ppb) level. This translates into very tight specs on beam quality, especially the degree of correlation between the helicity state and beam parameters, such as position, angle or intensity. These are referred to as helicity-correlated (HC) parameters. Table 1 gives an overview of the intrinsic physics asymmetries and requirements on HC beam properties by various experiments at Jefferson Lab., two of which, HAPPEX-II

Table 1: Specs on Experiment-Averaged HC Parameters

\begin{tabular}{|c|c|c|c|c|}
\hline Experiment & $\begin{array}{c}\text { Physics } \\
\text { Asym. } \\
\text { (ppm) }\end{array}$ & $\begin{array}{c}\text { Intensity } \\
(\mathrm{ppm})\end{array}$ & $\begin{array}{c}\text { Position } \\
\text { on Target } \\
\text { (nm) }\end{array}$ & $\begin{array}{c}\text { Angle on } \\
\text { Target } \\
\text { (nrad) }\end{array}$ \\
\hline HAPPEX-I & 13 & 1.0 & 10 & 10 \\
\hline G0 & $2-50$ & 1.0 & 20 & 2 \\
\hline HAPPEX-He & 8 & 0.6 & 3 & 3 \\
\hline HAPPEX-II & 1.3 & 0.6 & 2 & 2 \\
\hline Qweak & 0.3 & 0.1 & 20 & 100 \\
\hline Lead & $<1$ & 0.1 & 1 & 1 \\
\hline
\end{tabular}

Hydrogen and G0 Backward Angle, were completed recently with all HC parameters within spec, partly due to significantly improved transport optics.

\section{ASYMMETRY AND HELICITY CORRELATED BEAM PARAMETERS}

The sub $100 \mathrm{ppb}$ level of precision requires measures by parity experiments to minimize both systematic and statistical errors. To this end parity experiments take advantage of the high beam current $(40-100 \mu \mathrm{A} \mathrm{CW})$ and high polarization ( $>70 \%$ ) available at CEBAF to enhance statistics. To reduce systematic errors, the following measures are adopted or in preparation:

- Beam helicity is flipped at $30 \mathrm{hz}$ to minimize contribution from slow systematics.

- Algorithm is implemented to subtract off false HC contributions by measurable beam parameters. 
- As all HC effects originate from the electron source, great efforts were devoted to reducing their source in the laser and photocathode systems [1].

- Active feedback can be applied as an option. Intensity feedback is a standard feature for PV experiments. Well-behaved empirical response is needed for effective orbit feedback.

HC orbits can be magnified by beam transport irregularities. To suppress such effects to the sub $100 \mathrm{ppb}$ level as mentioned, we need to maintain very high transport quality throughout the entire beamline.

In the interest of the current report, we will be mainly focused on the last bullet. The first 4 will be addressed to the extent they are relevant.

\section{Source of Helicity Correlated Parameters}

As indicated in Figure 2, a gross simplification of the concept of helicity flipping, polarization of electron is generated by a circularly polarized laser light incident on a photocathode, which produces electrons with positive or negative longitudinal spin states depending on the left or right handedness of the light polarization. The latter in turn is determined by the Pockels cell voltage polarity when the original linearly polarized light passes through it. By switching the Pockels cell voltage in $33.3 \mathrm{~ms}$ windows, we create pairs of 33.3 windows of longitudinal polarization in the electron beam.

Figure 2: Helicity Flipping by Pockels Cell

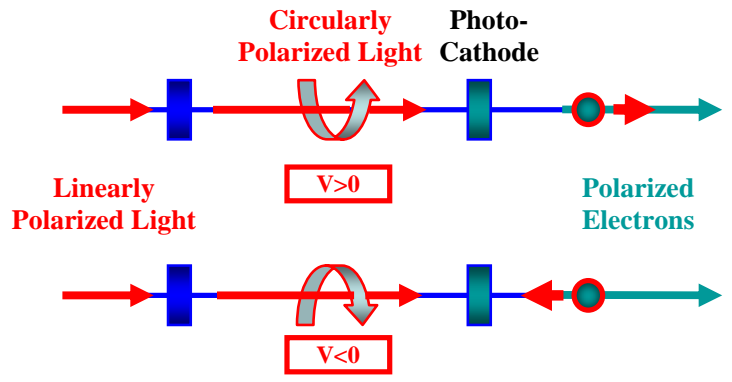

Any linear component in the circularly polarized laser due to imperfection causes asymmetric transmission and electron production at the photocathode for different helicities. This leads to helicity-correlated intensity. Another source for $\mathrm{H}-\mathrm{C}$ intensity can come from scraping at tight apertures by beam displaying nonzero $\mathrm{H}-\mathrm{C}$ orbits.

Whatever effect can produce $\mathrm{H}-\mathrm{C}$ intensity can also produce $\mathrm{H}-\mathrm{C}$ orbit, by shifting the beam centroid, if such effect has a non-zero gradient across the laser/beam profile. Thus if a gradient exists either in the linear polarization component of the laser, or in the analyzing power of the photocathode, $\mathrm{H}-\mathrm{C}$ orbit could emerge.

Finally the Pockels cell can act as a lens, focusing or defocusing laser light depending on the voltage applied. If it is not well aligned with respect to the light axis, it can create a steering effect and thus $\mathrm{H}-\mathrm{C}$ orbit.

Significant effort has been devoted, with major success, to the minimization of these sources in the CEBAF Injector [1]. The result has contributed in part to the recent success in meeting all parity experiment specs.

\section{Correction of Raw Asymmetry}

The asymmetry $\boldsymbol{A}_{P V}$ of equation (1), measured directly by comparing cross sections from left and right handed electron states, must be subjected to correction to subtract off false contribution due to its dependence on beam systematics, such as intensity, energy, position and angle. This process is described as

$$
\begin{aligned}
A_{P V} & =A_{D E T}-A_{Q}-\alpha A_{E}-\sum_{i} \beta_{i} \cdot \Delta X_{i} \\
\beta_{i} & =\frac{\partial A}{\partial \Delta X_{i}}, \alpha=\frac{\partial A}{\partial \Delta E}
\end{aligned}
$$

where $\boldsymbol{A}_{D E T}$ is the raw asymmetry, $\boldsymbol{A}_{Q}$ the asymmetry due to intensity fluctuation, $\alpha$ and $\beta$ are empirical sensitivity coefficients linking beam energy asymmetry $\boldsymbol{A}_{E}$ and helicity correlated variations in position (angle) $\triangle X$ to the detector asymmetry.

The empirical coefficients $\alpha$ and $\beta$ inevitably carry errors. In the absence of helicity correlated beam parameters, such errors will average out with large statistics of data from both helicity states. On the other hand, helicity correlated beam parameters will cause such errors to be retained in the final corrected asymmetry. It is this correction procedure that defines the allowable helicity correlated beam parameters. For example for HAPPEX-II experiments, given desired precision (5\%) on the asymmetry (1 ppm), and estimates on the uncertainties $(10 \%)$ in $\beta x$ and $\beta x^{\prime}$, the allowed $\mathrm{H}-\mathrm{C}$ position and angle averaged over a data acquisition run are limited to values given in Table 1.

\section{MEASURES TO CONTROL HELICITY CORRELATED BEAM PARAMETERS}

Besides efforts to fine-tune the laser system to minimize sources of HC parameters as mentioned, the current report will mainly focus on complementary work done to minimize transport anomalies that can disrupt the natural reduction in $\mathrm{HC}$ orbit amplitudes due to momentum increase from the cathode (335 keV/C) to the target ( $3 \mathrm{GeV} / \mathrm{C})$, known as adiabatic damping. If the entire beam path is governed by well-behaved optics free of XY coupling, the amplitude of any HC orbit should reduce by almost a factor of 100 when it reaches target. In the presence of transport anomalies, this factor can be compromised to almost arbitrary degree, especially when XY coupling and near singular transport are compounded.

\section{TRANSPORT ANOMALIES}

\section{XY Coupling}

$\mathrm{XY}$ coupling can potentially introduce projected emittance growth. Without a correction system that can handle full 4D phase space, this growth cannot be undone.

\section{Near Singular Transport}

Transport can be near singular when it leads to excessive correlation between independent coordinates, to the degree that they are effectively no longer independent for given precision of measurement and control. They are 
undesirable for the following reasons.

- Excessive increase in projected coordinates

- Extreme demand on accuracy to measure or control

- Extreme sensitivity to minor perturbations

- Projected emittance growth by (otherwise benign) optical errors

The last two points are why such anomalies should be fixed as early as possible in a beamline. The simplest model for projected emittance growth of an initially uncoupled beam through a skew quad of strength $\boldsymbol{k}$ is

$$
\varepsilon_{X \text { Final }}^{2}-\varepsilon_{X \text { Inital }}^{2}=\beta_{X} \beta_{Y} \varepsilon_{X} \varepsilon_{Y} k^{2}
$$

showing the damage that can be done by a grossly mismatched beam in the presence of XY coupling. Such combinations were observed in two areas in CEBAF as the dominant cause for missing damping of HC orbits.

\section{FIXING TRANSPORT ANOMALIES IN CEBAF INJECTOR}

\section{Overall Strategy}

Transport anomaly in the form of compounded coupling and near-singularity appears in two main forms in the CEBAF Injector. Depending on the beamline extent and tools, different plans were formulated.

- From $100 \mathrm{keV}$ (K.E.) to $60 \mathrm{MeV}$, with accurately measured transport and sufficient correction elements, a model-based solution is possible.

- From $60 \mathrm{MeV}$ to $3 \mathrm{GeV}$, lacking accurate long-range model, an empirical approach appears more practical.

\section{$100 \mathrm{keV}$ to $60 \mathrm{MeV}$}

The first sign of transport anomaly in this area was seen when propagation of spot motion on the cathode, induced by piezo-electric transducer (PZT) driven mirrors, was observed. Figure 3 shows the momentum-normalized propagation of $\mathrm{X}$ and $\mathrm{Y}$ motions induced by PZT across the Injector. A schematic of the Injector is given in Figure 4 showing the SRF components: one cryo-unit and

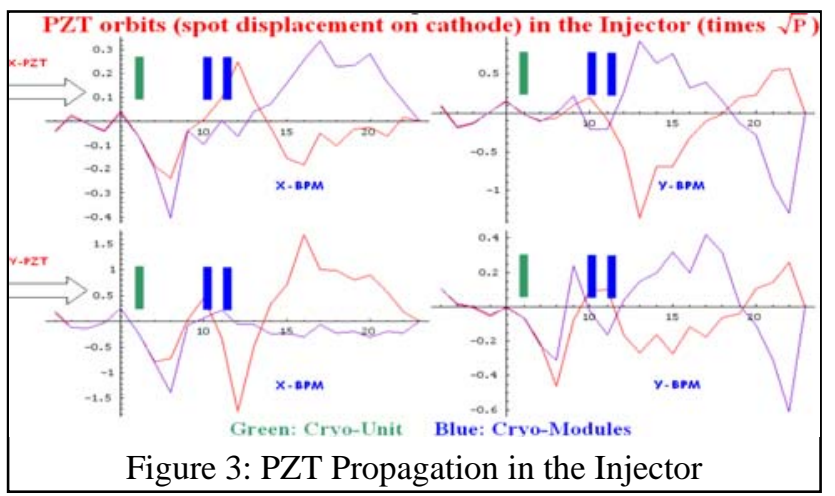

Figure 3: PZT Propagation in the Injector two cryomodules. The HOM couplers in these SRF units are known to introduce XY coupling. This, together with near singular transport due to imperfect modeling at low energy, resulted in phase space blowup represented by the amplitude jumps across these units in Figure 3.

Four-dimensional (X, X', Y, Y') transfer matrices were measured using difference orbit methods across these units. This proved challenging due to high precision required one the one hand, and compromising factors on the other, such as degenerate optics at $100 \mathrm{keV}$, limitation to orbit amplitude due to aperture and aberration, limited BPM's and noisy signal. Having overcome most of these difficulties, we were able to obtain 4D transfer matrices across the combined capture+cryo-unit and across each of the cryomodules. Figures 4 and 5 show the performance of these empirically measured $4 \mathrm{D}$ transfer matrices. For all trajectories launched before the unknown transport of interest, with a good coverage of initial phase space, these plots show comparisons between the exit coordinates predicted by the empirical matrices (blue) and those actual measured (red). The measured empirical matrices are

$$
\left(\begin{array}{llll}
3.304 & 0.526 & 1.237 & 0.251 \\
1.896 & 0.32 & 0.571 & 0.115 \\
0.894 & 0.183 & 2.066 & 0.309 \\
0.109 & 0.021 & 0.765 & 0.144
\end{array}\right) \quad\left(\begin{array}{llll}
-1.958 & 2.034 & 0.147 & 0.916 \\
-0.101 & 0.052 & 0.014 & 0.049 \\
-0.075 & 0.503 & -0.839 & 0.442 \\
0.011 & -0.005 & -0.032 & -0.108
\end{array}\right)
$$

respectively. It is worth noting that

- These matrices explain the transport very well.

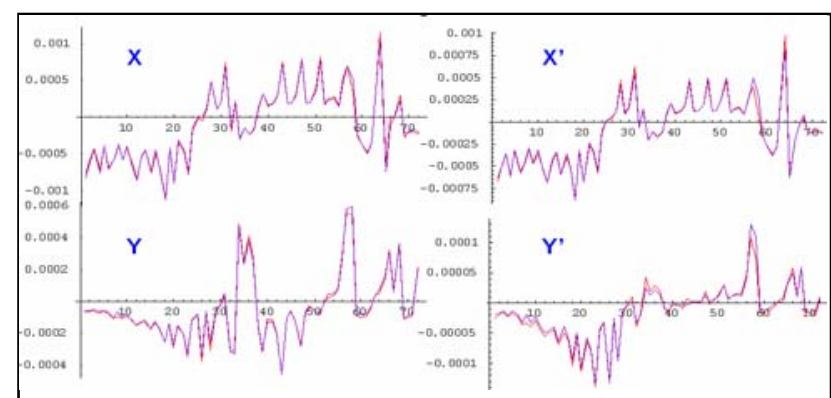

Figure 4: Coordinates Measured (red) vs Predicted (blue) by Empirical Matrix at Exit of Cryo-unit for 72 Orbits.

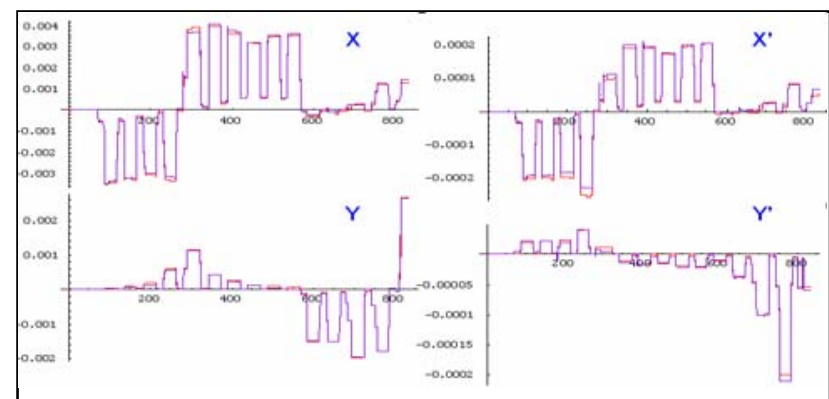

Figure 5: Coordinates Measured (red) vs Predicted (blue) at Exit of Cryo-module for 850 Orbits.

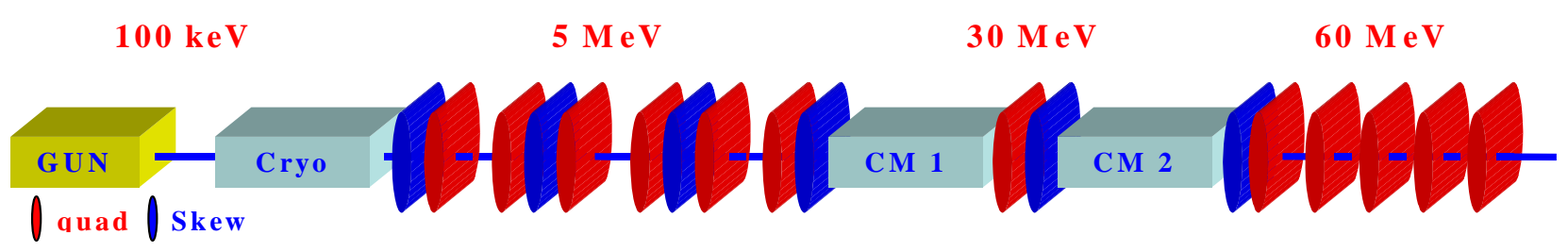

Figure 4: CEBAF Injector $100 \mathrm{keV}$ to $60 \mathrm{MeV}$ 
- They display significant singularity, as can be seen either in the absence of damping in the elements, or in the near degenerate patterns in the exit X \& X'.

- They both satisfy the linear 4D symplectic condition exactly, implying that only linear effects are at work, $4 \mathrm{D}$ phase space is preserved, and proper transport can be restored with linear elements only.

An optimization program was developed with the aim of using the existing quadrupoles and skew quadrupoles to eliminate $\mathrm{XY}$ coupling and minimize singularity from $100 \mathrm{keV}$ to $60 \mathrm{MeV}$. This seemingly straightforward task was complicated by additional criteria that the solution must respect. These include compatibility of transported beam and PZT orbit with downstream acceptance, impact on optics needed for feedback system, beam size, and quad/skew quad strengths due to field quality and orbit concerns. These criteria are listed in Table 2. Under these multitude of constraints solutions were obtained via a combined global-local approach:

- Search in parameter space within magnet range to map out solution landscape,

- Cutoffs based on multiple constraints to isolate viable solution neighborhoods,

- Local optimization to reach respective global optima.

Measurements of PZT orbits, beam profile, and 4D transfer matrix encompassing the capture cavity, cryo-unit and both cryomodules were input to this program. A solution was implemented before transfer same matrix was measured again. These two matrices are

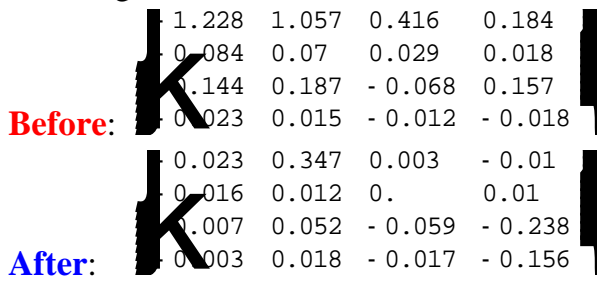

While both are $4 \mathrm{D}$-symplectic, their transport properties are widely disparate. This is summarized in Table 3 . The solution has eliminated XY coupling (off-diagonal determinant as percent of total), and brought degeneracy (SVD condition number) in the original transport down to near-ideal level, while respecting all criteria of Table 2.

\section{$60 \mathrm{MeV}$ to $3 \mathrm{GeV}$}

It was clear from early on that the PZT orbit undergoes amplitude increase as it goes from the Injector $(60 \mathrm{MeV})$
Table 3: Margin Specifications

\begin{tabular}{|l|c|l|l|}
\hline 2005 Beam Based Data & Ideal & Before & After \\
\hline \% Off-Diag. Determinant & 0 & 40.67 & 0.518 \\
\hline X-Sub Matrix Cond. No. & $\sim 10$ & 863.79 & 23.62 \\
\hline Y-Sub Matrix Cond. No. & $\sim 10$ & 9.651 & 16.50 \\
\hline 4 X 4 Matrix Cond. No. & $\sim 10$ & 562.68 & 25.55 \\
\hline
\end{tabular}

to the main accelerator. This increase takes place in the section up to $200 \mathrm{MeV}$ in the first linac. This is mostly due to imperfect model, field cross talk, and inaccurate linac energy profile. It is more betatron mismatch than $\mathrm{XY}$ coupling. However, as discussed above, this can be fertile ground for projected emittance growth given the right amount of otherwise harmless XY coupling. The two linacs of CEBAF consist of 20 cryomodules each, with the same HOM-induced coupling. Skew quads have been installed near each cryomodule for local compensation. Beam based tests [2] nonetheless suggest that residual coupling can be uncertain up to $20 \%$. This is a systematic effect and can add coherently. It is thus interesting to examine how a betatron mismatch can exacerbate the effect of such coupling. It is important to note that helicity-correlated orbits are typically 10-100 times smaller than spot size, and therefore can be much more mismatched to design optics without causing beam loss. This hidden problem can thus develop undetected and present a challenge to later attempts to control it.

Simulation was carried out to quantify this effect. Different amounts of betatron mismatch were introduced in matching from the Injector into the main accelerator, followed by 5 -pass propagation in the presence of said residual coupling. Two parameters were examined.

We first look at the projected emittance (Fig. 6a). For well-matched beam $(\mathrm{CS}=1)$, even in the presence of residual coupling, this barely changes. But if $\mathrm{CS}=5$, the projected emittance can be as large as 30 times larger, and incidentally happens at $3 \mathrm{GeV}$, the energy for parity experiments. We next look at the beam-orbit mismatch factor (Fig. 6b). Again if the initial matching is good, initially congruent beam and orbit will stay close on the same phase ellipse, as indicated by mismatch factor close to 1 throughout. This can grow from 1 to as large as 7 at $3 \mathrm{GeV}$ if the initial mismatch is large, however. 
The empirical approach adopted for correcting mismatch from $60 \mathrm{MeV}$ to $3 \mathrm{GeV}$ is betatron matching under the guidance of the $\mathrm{X}$ and $\mathrm{Y}$ PZT signals, as they define a subspace in the $4 \mathrm{D}$ phase space shown to be representative of the characteristics of true $\mathrm{HC}$ orbits. This has proved effective, with the exception that in some cases when the PZT orbits are not sufficiently congruent with the beam spot itself, in which case a compromise must be empirically reached.
Injector was corrected. Figure 8 shows the same seen at $3 \mathrm{GeV}$ in experimental Hall A (HAPPEX). The HC orbit reduction from cathode to target was significant. This translated into unprecedented $\mathrm{HC}$ orbit reported by HAPPEX 2005 at $3 \mathrm{GeV}$, as shown in Figure 9a and by G0 at a much lower energy of $650 \mathrm{MeV}$ (more demanding for similar parity experiment specs) in Figure 9b.

\section{CONCLUSION}

We discussed efforts devoted to identifying anomalous transport in the low energy section of CEBAF, and techniques developed to correct them. This is seen to result in unprecedented helicity correlated orbit, partly

Table 2: Criteria in Effect for Finding Optimal Injector Matching Solution

\begin{tabular}{|l|l|l|l|}
\hline Property & Figure of Merit & Goal/Limit & Comment \\
\hline Decoupled transport $100 \mathrm{keV}$ to $60 \mathrm{MeV}$ & Off-diagonal elements in 4D matrix & 0 & On paper this is not too difficult. \\
\hline $\begin{array}{l}\text { Non-singular transport } 100 \mathrm{keV} \text { to } 60 \\
\mathrm{MeV}\end{array}$ & $\begin{array}{l}\text { SVD condition number X/Y } \\
\text { M11/M33: measure of singularity }\end{array}$ & $\begin{array}{l}\text { SVD: } 250 \\
\text { M11/M33: } 1.0\end{array}$ & \\
\hline PZT-defined Twiss matched to $60 \mathrm{MeV}$ & Courant Snyder mismatch parameter X/Y & 10 & Advisory rather than absolute \\
\hline $\begin{array}{l}\text { PZT amplitude reduction } 100 \mathrm{keV} \text { to } 60 \\
\mathrm{MeV}\end{array}$ & Amplitude peak \& RMS & $\begin{array}{l}\text { Peak: }>10 \\
\text { RMS: }>10\end{array}$ & Advisory rather than absolute \\
\hline Spot size from $5 \mathrm{MeV}$ to $60 \mathrm{MeV}$ & Spot size everywhere & TBD & \\
\hline Match of beam spot into $60 \mathrm{MeV}$ line & Courant Snyder mismatch parameter X/Y & 10 & Advisory rather than absolute \\
\hline Skew quad strength & Field integral & $\begin{array}{l}5 \mathrm{MeV}:<20 \mathrm{G} \\
\text { All else: }<40 \mathrm{G}\end{array}$ & $\begin{array}{l}\text { To avoid inaccuracy and } \\
\text { misalignment kicks }\end{array}$ \\
\hline Quad strength & Field integral & Physical limit & \\
\hline $\begin{array}{l}\text { Impact on } 5 \mathrm{MeV} \text { helicity feedback } \\
\text { system functionality }\end{array}$ & $\begin{array}{l}\text { SVD condition number of 4D response from } \\
\text { feedback magnets to downstream }\end{array}$ & $\begin{array}{l}<500 \text { scaled } \\
\text { by M12/M34 }\end{array}$ & $\begin{array}{l}\text { Helicity feedback system needs } \\
\text { good 4D phase space coverage }\end{array}$ \\
\hline
\end{tabular}

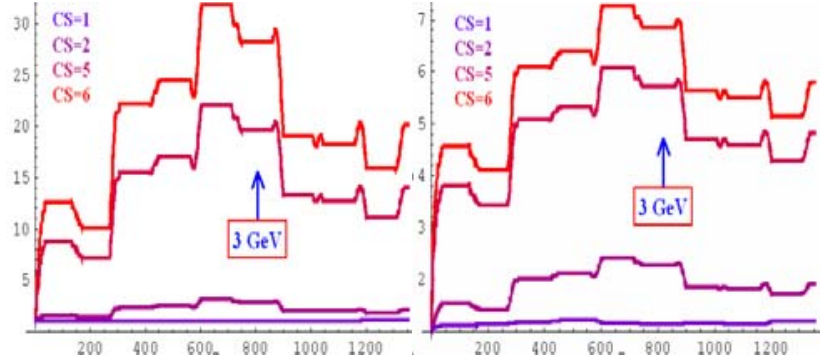

Figure 6: (a) Left: Projected Emittance; (b) Right: BeamOrbit Mismatch for Various Initial Mismatch Propagated through 5 Pass CEBAF

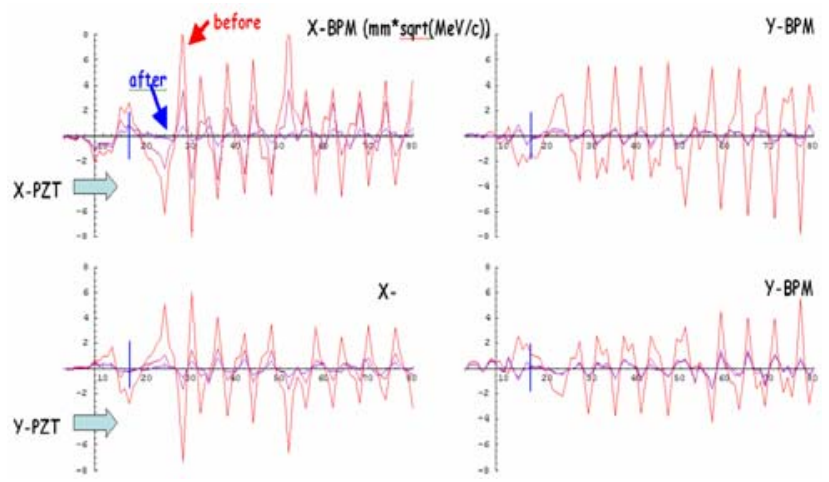

Figure 7: PZT response before and after Injector Matching from $100 \mathrm{keV}$ to $800 \mathrm{MeV}$

\section{OUTCOME OF INJECTOR MATCHING}

Figure 7 shows the response at the BPM's, from 100 $\mathrm{keV}$ to $800 \mathrm{MeV}$, to the X and Y PZT's before (red) and after (blue) the anomalous transport in the CEBAF responsible for the first JLab parity experiment to achieve asymmetry measurements precision better than $100 \mathrm{ppb}$.

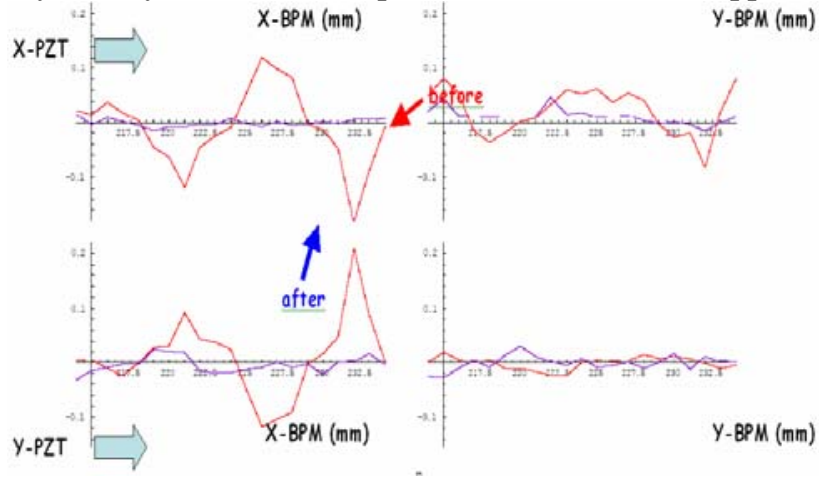

Figure 8: PZT response before and after Injector Matching at $3 \mathrm{GeV}$ in Hall A 


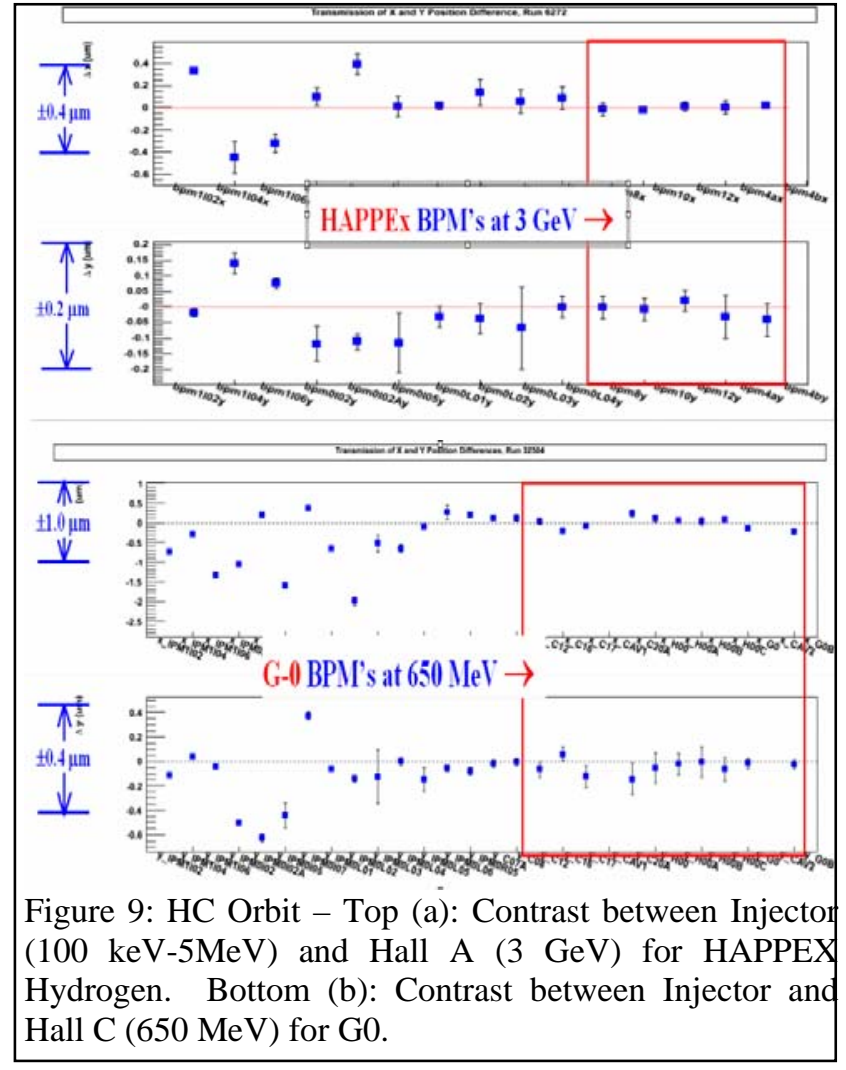

\section{ACKNOWLEDGEMENT}

Work by the following people are critical in achieving high quality beam at CEBAF as reported here: H. Areti, D. Armstrong, S. Bailey, D. Beck, J. Benesch, B. Bevins, A. Bogacz, S. Chattopadhyay, R. Dickson, A. Freyberger, J. Grames, J. Hansknecht, A. Hutton, M. Joyce, L. Kaufman, R. Kazimi, K. Nakahara, K. Paschke, M. Pitt, M. Poelker, Y. Roblin, M. Spata, R. Suleiman, M. Tiefenback, Y. Zhang.

\section{REFERENCES}

[1] K. Paschke, "Controlling Helicity-Correlated Asymmetries in a Polarized Electron Beam", PAVI06, Milos, May 20, 2006.

[2] Y. Chao, "Linac Skew Quad Calibration", July 2003, http://www.jlab.org/ chao/Skew_quad_calib.pdf. 